\title{
Experimenting with Design Thinking in Requirements Refinement for a Learning Management System
}

\author{
Marcos P. da Soledade Jr' ${ }^{1,2}$, Ricardo S. e Freitas ${ }^{2}$ \\ Sarajane M. Peres ${ }^{2}$, Marcelo Fantinato ${ }^{2}$, Reinhold Steinbeck ${ }^{3}$, Ulisses F. Araújo ${ }^{2}$ \\ ${ }^{1}$ Quantica TI e Comunicação - Social and Tecnologic Incubator of University of São Paulo \\ São Paulo - SP - Brazil \\ ${ }^{2}$ School of Arts, Science and Humanities, University of São Paulo (USP) \\ São Paulo - SP - Brazil \\ ${ }^{3}$ Center for Design Research, Stanford University \\ Stanford - CA - USA \\ m.soledadedquanti.ca, steinbeckestanford.edu, \\ \{ricardo.freitas, sarajane, m. fantinato, uliarau\}@usp.br
}

\begin{abstract}
In the information systems development, software engineers seek to improve the systems' functionality and usability, which may require a review of their requirements. Considering a teaching-learning process scenario, guaranteeing such goals demands to comply with pedagogical rules and satisfy student desires and needs. This paper reports on an experiment in requirements refinement for a Learning Management System, involving the system's users through the application of the Design Thinking methodology. Design Thinking is a human-centered set of techniques and tools that supports an iterative process to produce, analytically and creatively, solutions for real challenges. Also, the authors present a set of refined requirements as a result of the experiment.
\end{abstract}

\section{Introduction}

Requirements elicitation is one of the first activities carried out during the Information Systems (IS) development, usually conducted by software engineers together with their customers. In the teaching-learning process supported by a Learning Management System (LMS), the requirements are commonly elicited with the program's pedagogical coordinator, not involving necessarily end users, i.e. the students. The lack of students in this process may lead to a system that, although able to meet functional requirements, fails in meeting non-functional requirements (e.g. usability ${ }^{1}$ ). Thus, in order to improve the requirements elicitation activity, considering mainly the usability characteristics, it is important to know some aspects related to end users' experience, such as emotional and cognitive features that can point out users' desires or questions.

This paper discusses an experiment whose objective was to meet a set of teachinglearning process needs assuming that the students' participation during the LMS development shall be taken as an effective strategy. In this context, to systematize the students' insertion to the LMS development process, the Design Thinking (DT) methodology was

\footnotetext{
${ }^{1}$ Usability must involve: appropriateness recognisability, learnability, operability, user error protection, user interface aesthetics and accessibility, as specified in ISO/IEC 25010:2011 guide.
} 
applied, so that the student was placed as an active agent in the design process of the solution, i.e. addressing the LMS requirements. The experiment was designed to let students feel free to express their preferences, desires, questions and ideas about the LMS. The system developers were responsible for refining the software requirements using the content produced by the students.

The goal of this paper is to describe the experiment, discuss the possibility of applying commonly used DT strategies in an IS development, and present the refined requirements for the LMS that resulted from this process. The paper is structured as follows: Sections 2 and 3 present, respectively, a brief discussion on DT definitions and the application of this and similar methodologies within the context of IS development tasks; Section 4 describes the experiment context, covering both the Virtual Learning Environment and learning circumstances in which it is used; the application of DT for requirements refinement is presented in Section 5, whereas the results, showing the refined requirements, are discussed in Section 6; final considerations are presented in Section 7.

\section{Design Thinking}

Design Thinking (DT) is frequently defined as a methodology for the development of innovative and useful solutions. Brown $(2008)^{2}$ defines DT as "a methodology that imbues the full spectrum of innovation activities with a human-centered design ethos." For Brown, it is about the use of designers' sensibility to address what is technologically appropriate and strategically viable when proposing a solution; DT allows (and does not discourage) the use of conventional and scientifically proven methods in problem-solving, but combining them with the designer's abilities to meet the people's needs. For Plattner et al. (2011), DT focuses on the end user, with multidisciplinary collaboration and interactive improvements. Moreover, Steinbeck (2011) points out that DT focuses on the design process rather than a product, i.e. something that can emerge from it.

According to Liu et al. (2011), DT refers to a mental process in which concepts are developed, decisions are taken and problems are solved, using expertise, knowledge and contextual information to satisfy certain goals (i.e., the design intents). Although there are several schools of thought and different definitions for DT, all of them gravitate to the same philosophy that covers creative thought, the contextual knowledge and scenarios in which a solution would be applied. An extensive review, with a great diversity of references regarding theories about DT, the philosophies that support them and application models, is presented by Liikkanen et al. (2011).

DT can be considered a universal methodology since it could be applied in any field of knowledge and to problems in any complexity level. Specifically for the IS development field, DT is studied from two perspectives: (a) considering that Information Technology (IT) and IS may support the application of DT and, in this sense, there are some efforts for the development of tools to provide such support [Uflacker et al. 2011; Gumienny et al. 2011; Sun and Liu 2009; Liu et al. 2011]; and (b) considering that the DT methodology can support problem-solving in the IT and IS contexts. The experiment discussed in this paper is contextualized in the second perspective.

\footnotetext{
${ }^{2} \mathrm{~T}$. Brown is CEO of IDEO - Sillicon Valley, one of the top ranking innovative companies in the world. He has already used his DT skills in companies such as Procter\&Gamble and Bank of America.
} 


\section{Related Works}

Several artifacts can be produced as DT outputs, such as policies, mindmaps, processes, or even solutions addressed to a specific scenario, such as requirements for a computer system or equations to an optimization problem. Nash et al. (2005) present, for example, the need of associating Software Engineering techniques to requirements elicitation and the software evaluation, regarding the needs of computer based teaching-learning process. These authors also point out a resemblance between system architecture's techniques and DT methodology, encouraging such combination. Examples of initiatives using DT in conjunction to system's project management, agile development and prototype-based programming are discussed by Plattner et al. (2011). And, in a line directly related to the DT work, Arantes (2011) presents an experiment in system requirements elicitation using participatory design, in which a website's end users assume an active role in the problem comprehension and in the solution proposition, i.e. the website's requirements elicitation.

Dynn et al. (2005) describe how the DT process may also be used for model refinements. Using the engineering classes context at Stanford University, KTH-Stockolm and the University of Uppsala (in Sweden), the current educational and pedagogical models were rethought to introduce DT in freshman years. The combination of DT and UCD (User-Centered Design), experimented by Gonzalez et al. (2010), illustrates another initiative in the education area. Students of Human Computer Interaction have applied DT with UCD into learning activities, projecting solutions related to software design. Moreover, Pasman and Wieringa (2011) discuss a collaboration initiative between a software company and a research institute, which aimed to support a UCD process. According to the authors, DT philosophy gradually introduced a new way of thinking for the participants, motivating activities in which they shared their perspectives and goals.

Jarvis et al. (2012) argue that it is useful and necessary to discuss the theories correlated to DT, analyze the results of the experiment and, most importantly, show details about the materials that are used/produced during DT sessions. In this work, the authors used a photo-essay format in order to describe the backstage of the DT sessions and the evidences about the prototype and solutions produced during the sessions. Arantes (2011) and Pasman and Wieringa (2011) also present in their works several illustrations showing alternative materials and activities used to express personal behavior changes or expression ideas, desires and questions - actions inherent to the DT methodologies. The experiment described in this paper is structured inspired by some of these works.

\section{Context of the Experiment: Blended Learning and the LMS}

The improvement and the wide applicability of distance learning have been embraced by educational institutions; however, its real effectiveness may only be achieved when political, pedagogical and technical forces work together. Taking this view, a special initiative, named Programa Univesp ${ }^{3}$ (Virtual University of São Paulo), was created in 2008 by the São Paulo State Government. It was conceived through a partnership among the State Secretariat of Economic, Scientific and Technological Development, three public universities (USP, Unicamp and Unesp ${ }^{4}$ ) and funding foundations. Recently, Univesp was transformed by a state law into the fourth state university of São Paulo.

\footnotetext{
${ }^{3}$ http://www.univesp.ensinosuperior.sp.gov.br/ (in Portuguese)

${ }^{4}$ http://www5.usp.br/en/; http://www.unicamp.br/unicamp/?language=en; http://www.unesp.br/eng
} 
Among the educational programs offered by Univesp, we highlight in this paper the lato sensu graduate programs ${ }^{5}$, which are managed by USP, and focus on those used for this experiment. These programs have applied a strategy based on a partial renunciation of the traditional teaching methods, mixing: traditional "live" classes, organized in centers geographically distributed across the State of São Paulo and mediated by tutors, with "distance" classes, supported by a LMS in which different types of Digital Learning Objects (DLO) are available. Examples of DLOs are video lectures produced by professors, media and TV producers and sign language speakers, as well as audio-text and digital animations. Moreover, through this LMS, students communicate to each others and to their tutors, take notes, develop some activities and are evaluated by the tutors, teachers and $a d$ hoc referees. The pedagogical model adopted in these programs follows the Problem-Based Learning approach [Bound and Feletti 1998].

Facing the need of offering a LMS compliant to such perspective, the programs' managers, in cooperation with IS professionals, raised a number of functional and nonfunctional requirements that should be fulfilled by the LMS (see an overview in Table 1). This LMS is based on the Moodle 2.2 platform, published under an open-source license. This platform supports user and group management tools, digital artifacts publishing, open discussion areas for users, message exchange system and adequate performance to support a program with approximately 1000 enrolled students as the case of the programs discussed here. Since these functionalities do not necessarily meet all the specific students', tutors' and managers' needs, this platform was customized and new components were developed to satisfy the system functional and non-functional requirements.

Table 1. Requirements elicited by managers and the technical team

\begin{tabular}{l}
\hline Functional requirements \\
\hline 1. Publish DLOs in a chronological sequence, organizing each class resource in separated areas, showing one class at a time. \\
2. Publish repositories where students are able to access all DLOs at once, in a consolidated view. \\
3. Allow that each student to take notes regarding every video published on the LMS. Those notes should be editable at any \\
moment, and the LMS should generate a report covering all the student notes. \\
4. Manage the students registration and split them in groups, linking each group to the respective tutor, and allow the \\
interaction between those students and between a student and a tutor, through private messages or public spaces. \\
5. Have an space to present general information about the program (schedules, bulletin boards, tutorials, etc.). \\
6. Manage the projects developed and submitted by the students, and have tools to enable collaborative work. \\
\hline \hline Non-Functional requirements \\
\hline \hline 7. DLOs should be displayed in logical but non-linear layout, allowing for intuitive and motivating access to them. \\
8. The user interaction with the LMS should be simple, avoiding improper use of it and students' demotivation. \\
9. All the system areas should follow a unique visual identity. \\
10. The visual design should be as pleasant as possible, avoiding the use of long texts and valuing the use of images. \\
11. The main LMS features should be properly displayed on mobile devices (smartphones and tablets). \\
\hline
\end{tabular}

By default, Moodle implements only the basic functional requirements requested by the managers, such as requirements 4 to 6 . The context of this paper is focused in the analysis of the other non-trivial requirements (i.e. functional requirements 1 to 3 and nonfunctional requirements 7-11), which needed to be implemented by the technical team.

The graphical interface of the LMS, specially developed for this program, does not follow the native architecture available in the Moodle platform. It heavily relies on the use of several images and dynamic elements, making use of "mosaics" to organize and link information, as illustrated in Figure 1. This design was mainly developed based

\footnotetext{
${ }^{5}$ Programs that provide a specialization in specific areas and take about two years. Generally, the degree awarded from such programs are graduate certificates or professional degrees.
} 


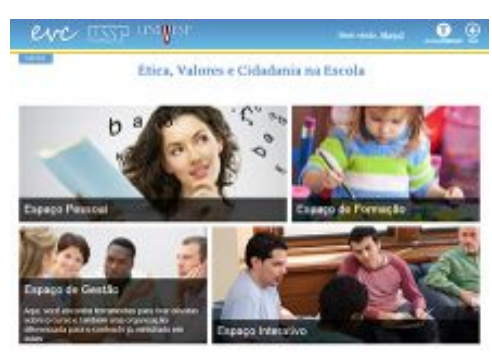

(a)

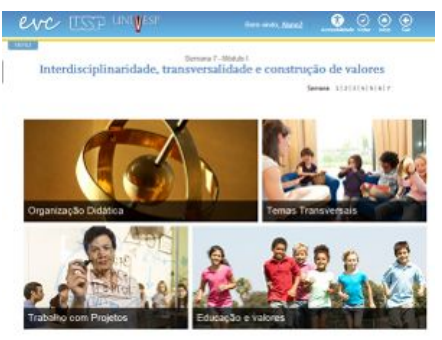

(b)

Figure 1. LMS Mosaics: (a) first screen on LMS - graphical menu to the system sections; (b) internal screen on LMS - graphical menu to access lecture contents.

on the non-functional requirements 7 and 11: the graphical elements have been arranged in order to improve the user access to the different system areas and to facilitate the adaptations needed for the implementation on mobile devices (considering touch-screen displays and smaller screen sizes), as illustrated in Figure 1. In this figure, two mosaics that have a menu function are presented: (a) a mosaic that links to four different sections in the platform (each section offers groups of specific functionalities); and (b) a mosaic that links to DLOs used in one of the distance-learning classes.

Requirements 1 and 2 demand different types of organization to the same content (the former is chronological, splitting content per classes, and the latter is grouped in the same space). Figure 2 shows the graphical interface to: (a) the space where weekly content (for each class) is presented; and (b) the space where all the media of a specific type of digital content (videos, in this case) is presented in a consolidated form. Figure 2(c) presents the video notes tool, which meets requirement 3 . These notes can be taken gradually, as long as the user studies the content, and can be edited at any time later. The students can also generate a report with their notes from all the videos.

The non-functional requirements 9 and 10 are those that motivated a DT session, given that a proper way to address all of them would require the system users' point of view, with each user expectations, personalities, preferences and familiarities with the LMS. Since the programs' managers pointed out the areas presented in Figure 2 as the ones most frequently used by the students, they were chosen as the first ones to be improved. Requirement 11 also was improved as a result of this initiative by enhancing the mobile devices support. The requirement 9 is constantly being sought by developers.

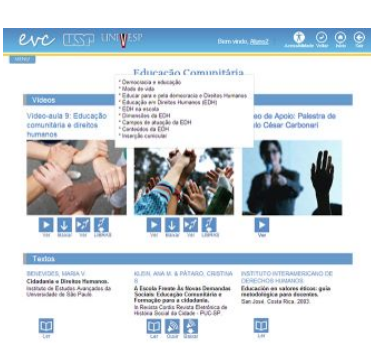

(a)

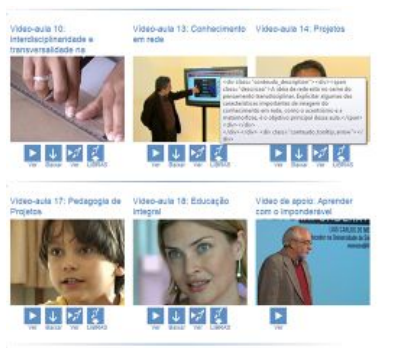

(b)

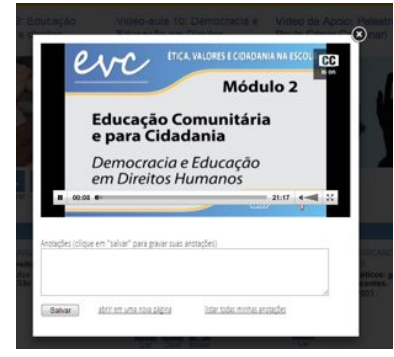

(c)

Figure 2. DLOs: (a) for a class; (b) the video library section; (c) video notes tool 


\section{Design Thinking Applied in the Requirements Review}

According to Brown (2008), design thinkers are people able to imagine the world under multiple perspectives and understand peculiarities of the environment, problems and solutions; they do not focus on problems when dealing with a challenge but see them as an opportunity, and they are collaborative work enthusiasts. According to Steinbeck (2011), DT processes strongly emphasize: (i) the acquisition of knowledge about the users of the product or the process being designed; (ii) the observation about the users to better understand their viewpoints and their reactions to the solutions proposals; and (iii) and the generation of as many ideas and solutions as possible. With this in mind, the design team conducted an experimental DT session for gathering and validation of ideas regarding the system scope. The objective of the DT session was to support the LMS requirements refinement, and thus maximize the success of the systemic solution launched to the users.

The session took place in October 2012, at the "Ideas Atelier" room of the Technological and Social Incubator of the University of São Paulo, and lasted 90 minutes. It was conducted by three IS professionals from the design team involved in the LMS development process; nine students with distinct undergraduate education and professional backgrounds ${ }^{6}$, participated in the session. All students were volunteers and were split to three groups with three students per group. While the session was being conducted, two of the IS professionals mediated the groups discussions, whereas the third IS professional acted as passive observer and noted information potentially useful to the experiment analysis. The DT session was suported by five main artifacts and actions: (i) the LMS itself, so that the users could use it and express their opinions; (ii) an environment (Figure 3(a)) specifically set up to stimulate the collaboration among the participants as well as their creative capacities (with colored papers, post-its, white board, colored pens, computers, internet access, besides proper furniture and other materials placed to provide comfort and informality); (iii) proper forms designed to guide the environment observer's analysis (Figure 3(b)); (iv) proper forms designed to stimulate communication among students (Figure 3(c)); (v) presentation of graphical interface mockups, illustrating implementations of some requirements (Figure 4) to work as a starting point for the session.

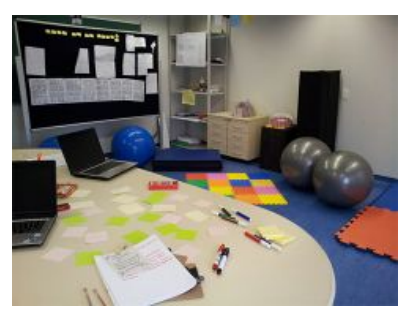

(a)

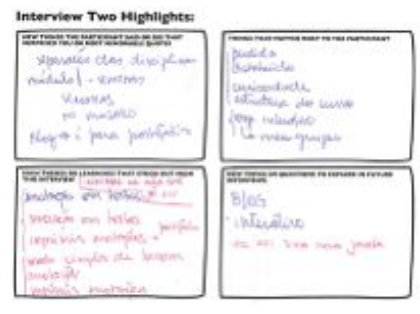

(b)

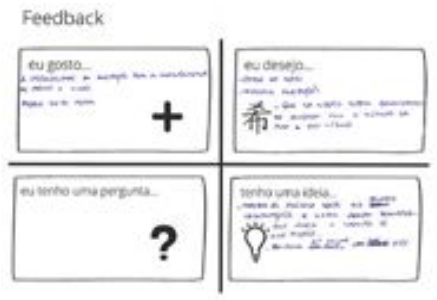

(c)

Figure 3. Artifacts used in the experiment: (a) physical environment; (b) interview forms' design; (c) feedback forms' design.

The interview forms were developed by John Nash ${ }^{7}$. They guide the DT session observation in order to provide a structured analysis in four areas: (a) surprising things

\footnotetext{
${ }^{6}$ This experiment was related to a program aimed to educators (primarily), social agents or education managements, among others.

${ }^{7}$ https://dschool.stanford.edu/groups/designresources/wiki/4dbb2/The_Wallet_Project.html
} 


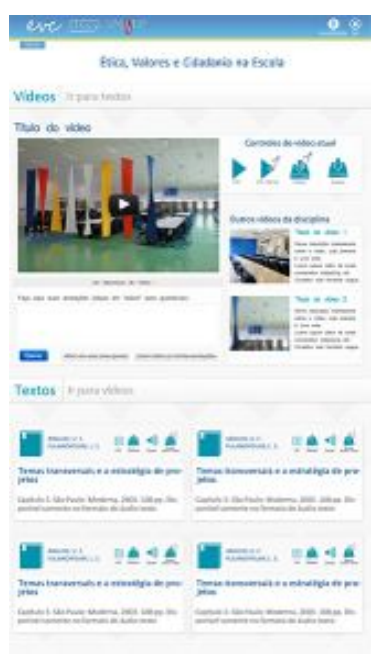

(a)

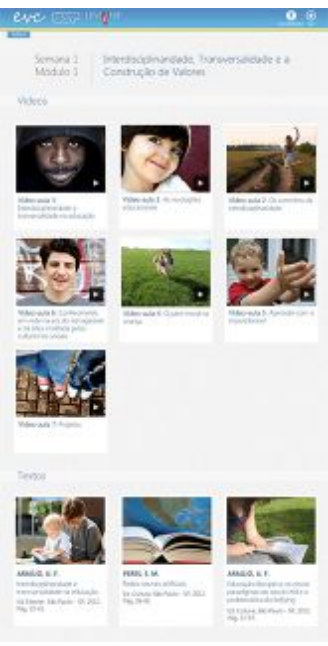

(b)

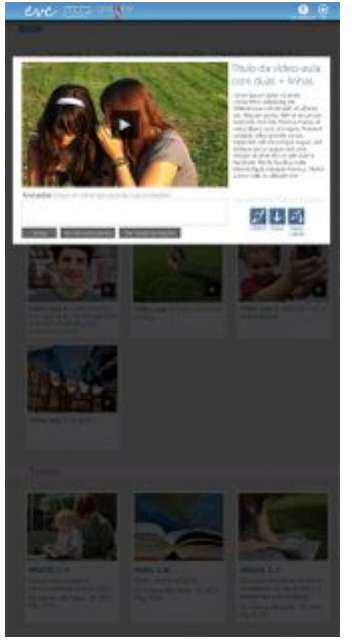

(c)

Figure 4. Mockups used in the experiment: (a) for a class; (b) the video library section; (c) video notes tool.

said by users; (b) what most concerns users; (c) learning derived from the interview; and (4) new topics or questions to explore in the future. Through this structure, mediators guided the groups interactions to focus on well structured objectives keeping such material as a support to organize their own observations, which is also useful for the observer.

The feedback forms ${ }^{8}$ were proposed by Reinhold Steinbeck, one of the co-authors of this paper. They are intended for making the interviewees explore their considerations about the session focus - in this case, the mockups (prototypes) of the LMS. This form aims at splitting the users' contribution in four segments: (a) I like; (b) I desire; (c) I have a question; and (d) I have an idea. Each one of them is intended to guide the users' feedback, preventing broad and superficial contributions, which would not express what they really feel or think about the system. Furthermore, the division between "desire" and "idea" segregates the "abstract" from the "concrete", allowing users to express desires that they do not have even a clue of how it can be satisfied and, at the same time, demanding them to look for solutions that can be quickly built. Moreover, the "question" creates a scenario in which users can criticize and show issues in the prototype, without feeling embarrassed by the presence of the technical agents that developed the system.

During the first part of the DT session, the three groups used the LMS and talked freely about the user interaction process. Two groups talked about ideas and experiences related to the LMS and to the program; whereas, the third group interacted with the system itself and exchanged information among them and the mediators. Occasionally, the groups' activities were switched, so that all students could express their impressions about the diverse system's aspects in more than one opportunity. During this part, only the interview forms were filled. During the second part of the DT session, the groups were merged and the mockups were projected onto the screen. Then, the feedback forms were distributed and the students could write their thoughts in the forms or express themselves verbally; while the mediators and the observer kept taking notes.

\footnotetext{
${ }^{8}$ http://dschool.stanford.edu/wp-content/themes/dschool/method-cards/i-like-i-wish-what-if.pdf
} 


\section{Results: Refining the Requeriments}

The main reason for conducting the DT session was to co-design the LMS requirements refinement process along with the users (i.e., the students). In order to present the results of such an experiment, this section is dedicated to report and discuss the opinions, desires, ideas and difficulties of the students, raised in the DT session, and the translation of these aspects into a refined set of system requirements. Moreover, this section presents the new designs for the system environments implemented once the DT session was carried out.

\subsection{Obtaining Data with Students}

After the DT session, data provided by students, through the forms and also through the observed interactions among themselves, were compiled by the mediators, the observer and other collaborators (co-authors of this paper). The data were then grouped by their similarities and analyzed according to the authors' perceptions. Table 2 lists the comments written by students, expressing their preferences, desires, questions and ideas. These data were obtained using the presentation of alternative mockups with possible changes proposed to the LMS graphical interface (see Figure 4).

Table 2. Students' comments. Comments' frequency are in parentheses.

\begin{tabular}{|l|l|l|}
\hline id. & Preferences & id. Desires \\
\hline 1 & Video abstracts beside the frame; not on top of it (7) & 14 Library organization per week (2) \\
2 & Text outside the picture; not upon it (2) & 15 Filenames of video-lessons as to their numbers and titles (2) \\
3 & Text within the picture; not outside it (1) & 16 No more only audio-texts (1) \\
4 & Images that relate to the video/text theme (2) & 17 Text editor with formatting features for notes (5) \\
5 & Titles of video files should be meaningful (1) & 18 Providing notes without having to open the video (1) \\
6 & Notes box below the video; not beside it (2) & 19 Option of generating pages with notes formatted for printing (3) \\
7 & Frames around texts in the library (1) & 20 Notes listed by course, week and video lesson (1) \\
8 & Choice of watching video in full screen (1) & 21 Space for notes on texts (2) \\
9 & Fewer windows for navigation (2) & \\
10 & New version more dynamic (1) & \\
11 & More organized and clean interface (1) & id. Ideas \\
12 & Beautiful, modern and dynamic visual (1) & 22 Place for talking to the tutor (1) \\
\hline id. & Questions & 23 Ability to mark videos/texts/weeks as already seen (1) \\
\hline 13 & Is there a better way to organize my notes? (1) & 24 Ability to download video-lessons in mp3 (for listening in the car, \\
& & for example) (1) \\
& & \\
\hline
\end{tabular}

Through an analysis on the students' comments, some issues regarding the LMS content disposition and the system interface could be realized. Comments 1 to 4 and 6 to 8 pointed out issues related to the GUI design. The complexity inherent to a graphical design can be seen when taking into account the diversity of opinions that sometimes leads to antagonic preferences (for example, the opposite preferences mentioned by comments 2 and 3). Comment 8 demonstrated that the video player design was not entirely appropriate, since users could not notice the already existing full-screen feature. Issues with the content organization were highlighted in comment 14 , in which the student requested an organizational structure not available in the LMS so far.

Indirectly, comments 4, 5, 7, 15 and 23 showed that students are demanding tools to allow them better manage the program environment and the DLOs. More specifically, comments 5 and 15 were concerned to the default file names when users download the resources. Comment 4 was a request for more comprehensive information regarding the DLOs' content. Comment 7 revealed a layout issue when presenting a specific DLO element (that is, the texts), once the students were not able to understand if a given icon 
was associated to the text above it or below it. Finally, comment 23 indicated that students needed more comprehensive support to manage their study process in the LMS. In addition to these, comment 9 was the only one related to the interface navigation: new browser windows need to be opened whenever the students visualize a PDF document or content hosted into an external website, which bothers users.

Comments 10 to 12 were related to the students' opinion on the new interface proposals, which were presented using the mockups (see Figure 4). These comments showed a good acceptance rate by the students. Moreover, the interface proposals met the students' preferences listed on comments 1, 3, 4, 6 and 7. Comments 13 and 17 to 21 were related to improvement suggestions on the video-note tool: the tool developed specially for this program, used to allow students to write notes about each video, while they watch it. The notes are linked to the video and are automatically retrieved when the students watch the video again. Comment 24 was related to an extra format for content delivery. Finally, comments 16 and 22 were related to decisions taken by the program's pedagogical responsible and, despite the impact on technical aspects of the LMS, their analysis are beyond the scope of this paper; although students are able to send messages through the Quickmail plugin, they are willing to have access to a synchronous communication tool.

The observations of the mediators and the observer also resulted in important information for the requirements refinement process seeing that they taken into account several types of communications from the students: students talking directly to the mediators; students talking to other students; and students thinking aloud. A summary about these observations is shown in Table $3^{9}$. In this table, the information is organized according to different LMS aspects. Considering the view of the mediators and the observer as system developers, the aspects observed were evaluated and classified in terms of the features and strategies already developed in the system. Among the observations listed in Table 3, it's worth mentioning two of them: 5 and 17. The former shows that during the DT session, there was a recurrent complaint about the lack of guidance regarding the use of DLOs published in the LMS. Nevertheless, a special DLO named "Didactic Organization" exists, in which such instructions are actually provided, published every week, as a part of the mosaic (Figure 1(b)), which seems to not be seen by students. The latter raises a possible issue concerning how much the platform (and the program as a whole) is succeeding in fostering the collaborative work once the tools that allow such work are placed in the Interactive Space section.

\subsection{Building the Refined Requirements List}

Supported by the information gathered during the DT session and described in the previous section, a new functional and non-functional requirements list was built. In this new list, some of the original requirements (as presented in Table 1) are refined and documented with a higher and additional detail level, focusing on the aspects that seem to be more important to the users. Table 4 shows the requirements refined. Only those requirements that have undergone through refinement are presented (i.e. requirements 1 , 5, 6, 9 and 10 are kept in their simple forms); for the other ones, one or more additional requirements with further details are presented.

\footnotetext{
${ }^{9}$ Some of these observations repeat the content of the students's comments (see Table 2) because some aspects were very commented during the DT session.
} 
Table 3. Mediators and observer's observations

\begin{tabular}{|c|c|c|}
\hline id. & Item & Observation \\
\hline 1 & Blogs & - There is no indication of the need to post a message \\
\hline 2 & & - Students do not know if what they post is used or not to evaluate them \\
\hline 3 & Mosaics & - Insufficient texts indicating what to do in the interface \\
\hline 4 & Navigation & - Bar indicating the current week number needs more emphasis \\
\hline 5 & & - There is no text indicating what to do with the DLOs of a course or the steps prior to a course \\
\hline 6 & & - There is no direct access to notes (the video associated with them need to be loaded) \\
\hline 7 & Videos & $\begin{array}{l}\text { - The layout of the DLOs in the GUI is indifferent to students (the most important is the clear organization } \\
\text { of them compared to last week/course/module) }\end{array}$ \\
\hline 8 & & - Students prefer watching streaming videos than after the download \\
\hline 9 & Texts & - Only audio-text upset students (there are materials supporting courses available only in audio) \\
\hline 10 & Library & $\begin{array}{l}\text { - Separation per week would really help students (this is a way of organizing content still nonexistent in the } \\
\text { LMS: or texts are associated to lessons or they are in a repository organized by courses) }\end{array}$ \\
\hline 11 & Notes & $\begin{array}{l}\text { - Students would like a more complete listing, with different possibilities of organization (per } \\
\text { module/week/video-lesson) }\end{array}$ \\
\hline 12 & & - Chance of formatting annotations with embedded text editor (a what you see is what you get editor) \\
\hline 13 & & - Ability to generate a consolidated and formatted page for printing notes \\
\hline 14 & General & - Possibility to mark, manually, a video lesson or a week as already visited \\
\hline 15 & & - Students fail to find a logical line that shows at what time of the program they are \\
\hline 16 & & - Students quite like the Documents and Tutorials area (a LMS page) \\
\hline 17 & & - Students do not understand what the purpose of the Interactive Space (a LMS section) \\
\hline
\end{tabular}

Table 4. Refined requirements

\begin{tabular}{|l|}
\hline Functional requirements \\
\hline \hline a - Publish repositories with different organizations (by week, by discipline, by module), where the students are able to \\
access all DLOs at once, in consolidated views. \\
3a - Provide a what you see is what you get editor for the notes tool. \\
3b - Provide different ways to organize and print the notes (by video, by week, by discipline, by module). \\
3c - Provide a notes tool for textual and audio DLOs. \\
4a - Improve the message tools to allow a direct talk (i.e. synchronous) between student and tutor. \\
\hline Non-Functional requirements \\
\hline 7a - Replace the use of video frames by images to indicate some information about the content of the videos. \\
7b - Improve the designing of the player's commands to indicate the possibility of watching a video in full screen. \\
7c - Highligth the DLO "Didactical Organization" in the graphical interface. \\
7d - Change the video files names (physical names). \\
7e - Provide a new format for DLOs (mp3). \\
8a - Highligth the DLOs already visited, in the graphical interface. \\
\hline
\end{tabular}

\subsection{New LMS Releases and Next Steps}

Once this DT session was carried out, some improvements were made into the LMS influenced by this experiment. Based on the mockups used in the DT session (presented in Figure 4), which were analyzed and approved by the students, the original DLOs presentation (as shown in Figure 3) were evolved to the new interface presented in Figure 5. In Figure 5(a) and (b), the fulfilment for the new requirement 7a can be observed, when comparared to the exhibited in Figure 3(a) and (b), respectively. Moreover, the new requirement $7 \mathrm{~d}$ was also met in the current LMS release.

Taking into account that a complex requirement from the original list (requirement 11 in Table 1) was still being developed during the three months since the DT session, the development team did still not have the opportunity to treat the other new requirements, besides the $7 \mathrm{a}$ and $7 \mathrm{~b}$. From now one, the development team will evaluate the new requirements still not implemented considering the technical feasibility and pedagogical suitability in order to plan the roadmap for next LMS releases.

Considering the new LMS releases, the development team intends to carry out new DT sessions aiming at evaluating the current release, including the versions specific for mobile devices (illustrated in Figure 6), as well involving students from other geographic 


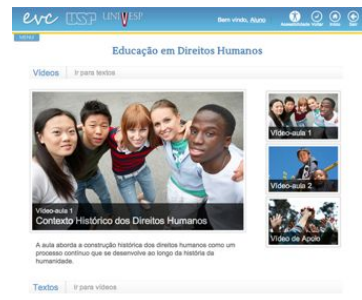

(a)

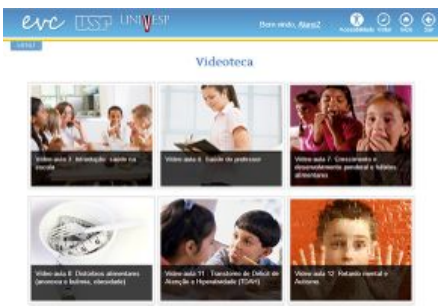

(b)

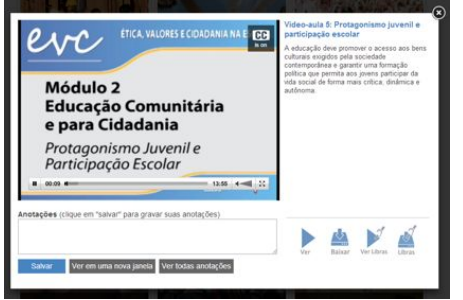

(c)

Figure 5. DLOs in the new LMS release: (a) for a class; (b) the video library section; (c) video notes tool.

locations and with a larger number of participants (both students and mediators).

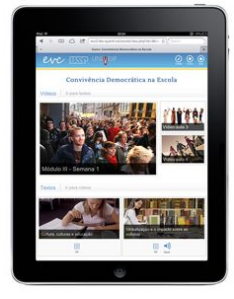

(a)

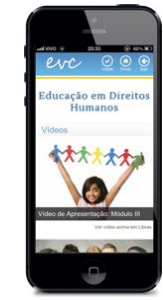

(b)

Figure 6. Mobile versions: (a) interface for tablets; (b) interface for smartphones

\section{Final Considerations}

The application of DT techniques to support the requirements refinement tasks in this context was considered appropriate. From this experiment, the authors could properly refine the requirements as well as learn from the system's users. An example of lesson learned was the detection of lack of alignment between the students' expectations and the pedagogical organization proposals. Another perception was that the developers invest significant time and resources implementing GUI details that are not noticed by the students, which was evidenced when variants of mockups with detail changes into the GUI (such as shadows, color tones and typographic elements) caused small impact on users. However, the DT session was carried out with few users. Thus, it is possible that there are other preferences, desires, questions and ideas which could generate other requirements.

Two research lines are planned in the context of this work: (i) building tools to analyse the students' navigation to gather information regarding the students' behavior on regular LMS use conditions; and (ii) integrating the LMS system with pedagogical models developed by the PBL Lab ${ }^{10}$ at Stanford University which provide communication channels for virtual brainstorming and collaborative 3D immersive virtual work spaces.

\section{Acknowledgments}

This work was supported by Center for Research on New Pedagogical Architectures (NPNAP) of University of São Paulo.

\footnotetext{
${ }^{10} \mathrm{http}: / / \mathrm{pbl}$. stanford.edu
} 


\section{References}

Arantes, F. L. (2011). Organizational semiotics and participatory design to requirements elicitation - a case study. In Proc. of 7th Braz. Symp. on Inform. System, pages 322-333.

Bound, D. and Feletti, G. (1998). The Challenge of Problem Based Learning. Routledge.

Brown, T. (2008). Design thinking. Harvard Business Review, 86(6):84-95.

Dynn, C. L., Agogino, A. M., Eris, O., Frey, D. D., and Leifer, L. J. (2005). Engineering design thinking, teaching, and learning. J. of Eng. Educ., 34(01):103-120.

Gonzalez, C., Gonzalez, E., Cruz, V., and Saavedra, J. (2010). Integrating the design thinking into the ucd's methodology. In IEEE Educ. Eng., pages 1477-1480.

Gumienny, R., Meinel, C., Gericke, L., Quasthoff, M., LoBue, P., and Willems, C. (2011). Design Thinking - Understand - Improve - Apply, chapter Tele-Boar: Enabling Efficient Collaboration In Digital Design Spaces Across Time and Distance, pages 147-164. Springer-Verlag.

Jarvis, N., Cameron, D., and Boucher, A. (2012). Attention to detail: annotations of a design process. In Proc. of the 7th Nordic Conf. on Human-Comp. Interaction: Making Sense Through Design, pages 11-20.

Liikkanen, L. A., Laakso, M., and Björklund, T. (2011). Foundations for studying creative design practices. In Proc. of the 2nd Conf. on Creativity and Innovation in Design, pages 309-315. ACM.

Liu, J., Zhang, M., and Hu, X. (2011). Dmml: A design thinking process modeling language. In Proc. of 12th Int. Conf. on Comp.-Aided Design and Comp. Graph., pages 44-48.

Nash, J. B., Richter, C., and Allert, H. (2005). Evaluating computer-supported learning initiatives. In Khosrow-Pour, M., editor, Encyclopedia of Information Science and Technology, pages 1125-1129. Idea Group.

Pasman, G. and Wieringa, E. (2011). Landing design thinking in industry: "making software for bookkeeping, but not in a bookkeeping way". In Proc. of the 2011 Conf. on Designing Pleasurable Products and Interfaces, pages 47:1-47:4.

Plattner, H., Meinel, C., and Leifer, L., editors (2011). Design Thinking - Understand Improve - Apply. Springer-Verlag.

Steinbeck, R. (2011). Building creative competence in globally distributed courses through design thinking. Scientific J. of Media Educ., 29(37):27-34.

Sun, Z. Gao, S. and Liu, J. (2009). A representation of context in design thinking process modeling. In Proc. of 11th Int. Conf. on Comp.-Aided Design and Comp. Graph., pages 416-421.

Uflacker, M., Kowark, T., and Zeier, A. (2011). Design Thinking - Understand - Improve Apply, chapter An Instrument for Real-Time Design Interactions Capture and Anlaysis, pages 131-146. Springer-Verlag. 